\title{
X-ray atomic mapping of quantum dots
}

\author{
Arka B. Dey $\odot,{ }^{1, *}$ Milan K. Sanyal $\odot,{ }^{1}$ Denis T. Keane, ${ }^{2}$ Gavin P. Campbell, ${ }^{2}$ Bo-Hong Liu, ${ }^{2}$ Ian Farrer $\odot,{ }^{3}$ \\ David A. Ritchie, ${ }^{4}$ and Michael J. Bedzyk ${ }^{2,5}$ \\ ${ }^{1}$ Surface Physics and Material Science Division, Saha Institute of Nuclear Physics, 1/AF Bidhannagar, Kolkata 700064, India \\ ${ }^{2}$ Department of Materials Science and Engineering, Northwestern University, Evanston, Illinois 60208, USA \\ ${ }^{3}$ Department of Electronic and Electrical Engineering, University of Sheffield, Mappin Street, Sheffield S1 3JD, United Kingdom \\ ${ }^{4}$ Department of Physics, University of Cambridge, J. J. Thomson Avenue, Cambridge CB3 OHE, United Kingdom \\ ${ }^{5}$ Department of Physics and Astronomy, Northwestern University, Evanston, Illinois 60208, USA
}

(Received 18 December 2019; revised manuscript received 17 March 2020; accepted 16 April 2020;

published 6 May 2020)

\begin{abstract}
We report a model-independent atomic-mapping technique for quantum dots (QDs) by combining Bragg reflection $\mathrm{x}$-ray standing wave (XSW) and grazing incidence diffraction (GID) measurements. In this study, we choose GaAs capped InGaAs QDs/GaAs(001) as a model system to show the locations and arrangements of indium atoms within the QDs along various $[h k l]$ directions. This technique directly reveals the actual amount of positional anisotropy and ordering fraction of indium atoms within the QDs by probing the (111), (111), (311), (131), (113), and (113) crystallographic planes. We find that indium atoms are outwardly shifted along the [001] direction by small fractions of the lattice constant, $0.04 a_{\mathrm{GaAs}}$ and $0.06 a_{\mathrm{GaAs}}$ from Ga sites for 50- and 150 - $\AA$ GaAs capped InGaAs QDs, respectively. We observe that an improved coherency factor of the indium atoms within the QDs by 45-60\% along the [001] and [011] directions reduces the photoluminescence linewidth by $22 \%$, thus making the QDs efficient for QD-laser and optoelectronic device applications. We also find that the position and ordering of In atoms along the (113) and (113) planes are most sensitive to the thickness of the GaAs cap layer. Our XSW-based results are supported by numerical calculations using a QD-macroscopic structural model based on our GID study. We thus show that this atomic-mapping technique will be useful for studying various quantum structures and tuning their properties.
\end{abstract}

DOI: 10.1103/PhysRevMaterials.4.056002

\section{INTRODUCTION}

Single layer quantum materials are predicted to deliver properties on demand [1] with interface engineering playing a decisive role in the tuning of structure-property correlation. Among these emerging materials, epitaxially grown quantum dots (QDs) confine electrons and holes in all directions giving atomlike energy levels and can thus deliver wavelengthtunable quantum photonic devices [2]. Tunability of photonic properties in QDs can be achieved by controlling segregation, intermixing, faceting, strain relief, and strain-enhanced diffusion $[3,4]$ during growth of these nanostructures. Understanding the structural morphology within QDs is essential for the growth of high quality QDs for device application. Atomic resolution measurements are required to develop such control of the desired growth of next generation photonic devices [2-4] such as large-area displays [5], QD-based laser diodes [6,7], and single-photon emitters [8,9]. Specialized nondestructive imaging methods [10] for quantum materials and buried nanostructures using resonant $\mathrm{x}$-ray-diffraction microscopy [11], coherent x-ray diffraction [12], and x-ray standing waves [13] are evolving to achieve such growth and property tuning in quantum materials.

\footnotetext{
*Present address: Deutsches Elecktronen-Synchrotron, Notkestraße 85, 22607, Hamburg, Germany; arka.bikash.dey@desy.de
}

The random spatial distribution of constituent atoms in self-assembled QDs create inhomogeneous broadening in optical emission spectra which limits the fabrication of photonic crystals and thus potential optoelectronic applications [14]. Anisotropy in the position and ordering of atoms has been detected indirectly through the measurement of optical properties. For example, Tahara et al. reported anisotropic optical properties of excitons for InGaAs QDs/GaAs(001) [15], and Cooke et al. reported anisotropic photoconductivity in InGaAs QDs chains [16]. Such anisotropic effects in optical, magnetic, and electronic properties have also been observed in GaAlAsQDs/GaAs(001) [17], CdSe-QDs/ZnSe [18], and other QD systems. However, it is still a challenge to deal with the anisotropic properties manifested by QDs as there is no study where the actual atomic-resolution positional anisotropy and ordering fraction of composite atoms are measured for QDs.

In previous studies [19], it has been shown that GID measurements can provide information about height, width, and overall shape of QDs, which are considered as key parameters for determining the positions of the photoluminescence (PL) lines. But the width of PL lines is solely dependent on the distribution of the ordered fraction of constituent atoms at coherent atomic position within the QDs. Here we report a method that combines two complementary synchrotron $\mathrm{X}$-ray measurements, namely grazing incidence diffraction (GID) and x-ray standing waves (XSWs), to obtain threedimensional (3D) atomic-scale maps of epitaxially grown 
buried layers of quantum dots. We measure the atomic positions and coherent ordering of these materials within an average unit cell which previously was completely lacking for QDs. We show that the distribution of lattice positions and ordering of constituent atoms within an average unit cell mainly in the [001] and [011] directions are essential for achieving sharp optical output widths which is important for QD lasers and single-photon emitter devices $[2,4,5,8,9]$. The ordered positions of atoms in an average unit cell of the QDs are directly measured by model independent XSW measurements and separately by indirect methods with numeric calculations from well established GID-obtained structural models on a QD-wide length scale for comparison. We studied two QD samples having different cap layer thicknesses. The capping layer is another important issue for practical application of QD based devices as it protects the QDs but changes their morphology [19]. In all previous studies only (002), (004), and (022) Bragg peaks were studied to understand the change in structure morphology within QDs with different cap layer thicknesses. In this study, we observe that (113) and (113) Bragg reflections are more sensitive to the cap layer thickness as the coherent ordering in those directions changes significantly when the cap layer thickness is increased to $150 \AA$ from $50 \AA$.

\section{EXPERIMENT}

We demonstrate this method using a GaAs capped single layer of indium gallium arsenide $\left(\operatorname{In}_{x} \mathrm{Ga}_{1-x} \mathrm{As}\right)$ QDs grown by molecular beam epitaxy (MBE) [20,21] under identical conditions on two separate GaAs (001) large wafer substrates. The details on the sample growth are given in the Supplemental Material (SM) [22]. Sample A is from the outer edge of the wafer with a 50 - $\AA$-thick GaAs capping layer grown by $\mathrm{MBE}$ and sample $B$ is from near the center of the other wafer with a 150- $\AA$-GaAs capping layer [3]. Total reflection X-ray fluorescence (TRXRF) analysis [23] using the indium $L \alpha$ XRF signal verifies the thicknesses of the cap layers and determines the In coverages to be 0.107 and $0.221 \mathrm{In} / \AA^{2}$ for samples A and B, respectively [22]. For the GaAs (001) surface one monolayer $(\mathrm{ML})=0.0625 \mathrm{Ga} / \AA^{2}$. The average heights of the QDs are found to be 340 and $310 \AA$, respectively, for sample A $(50-\AA$ cap) and sample B (150- $\AA$ cap) from GID measurements. Cross-sectional transmission electron microscopy (XTEM) analyses were performed to characterize the uncapped and capped QDs by using a FEI Helios NanoLab focused ion beam (FIB) and a field-emission JEOL 2100 FS/TEM operated at $200 \mathrm{kV}$ (refer to Fig. S2 in the SM [22]). Atomic-force microscopy (AFM) in tapping mode with a Nanoscope-IV multimode SPM was used to obtain average structural information of the uncapped QDs (refer to Fig. S3 in the SM [22]) by performing measurements on over 100 QDs. The XSW measurements were performed with an $8-\mathrm{keV}$ x-ray beam at the DND-CAT 5ID-C experimental station of the Advanced Photon Source, USA. (See setup in Fig. S1 in the SM [22].) The diffracted $\mathrm{x}$-ray beam intensity and indium $L \alpha$ fluorescence yield are collected simultaneously at each angular step by scanning in angle through the probed substrate GaAs Bragg reflections. High-quality GaAs(001) single crystals were used for growing the QDs as required for XSW measurements.
The GID measurements were carried out with a $25-\mathrm{keV}$ x-ray beam at beamline P08 of the Petra-III synchrotron in DESY, Germany. Photoluminescence (PL) measurements [24] were performed at a temperature of $4 \mathrm{~K}$ temperature with a laser source of wavelength $780 \mathrm{~nm}$.

\section{RESULTS AND DISCUSSIONS}

The model-independent, XSW measured $H=h k l$ coherent position $\left(P_{\mathrm{H}}\right)$ and coherent fraction $\left(f_{\mathrm{H}}\right)$ that sense the relative position and the spread in the spatial distribution of indium atoms respectively with respect to the unstrained $\mathrm{GaAs}$ substrate lattice can be related to the moduli and phase of the Fourier summation $F(l, n)$ of the In sublayers with ordered fractions $\left(s_{\mathrm{j}}\right)$ and positions $\left(p_{\mathrm{j}}\right)$ as

$$
\begin{aligned}
& f_{\mathrm{H}}=|F(l, n)| \exp \left[-2\left(\frac{\pi \sigma}{a_{\mathrm{GaAs}}}\right)^{2}\left(h^{2}+k^{2}+l^{2}\right)\right] \\
& P_{\mathrm{H}}=(2 \pi)^{-1} \operatorname{Arg}|F(l, n)|
\end{aligned}
$$

where

$$
|F(l, n)|=\sum_{\mathrm{j}=1}^{n} s_{\mathrm{j}} \exp \left(2 \pi i l p_{\mathrm{j}}\right) ;
$$

$n$ is the total number of sublayers. The exponential factor in $f_{\mathrm{H}}$ is the Debye-Waller factor that represents partially thermal and partially static disorder. $\sigma$ is found to be $0.2 \AA$. $a_{\mathrm{GaAs}}=$ $5.656 \AA$ is the GaAs unit-cell lattice parameter.

\section{A. Indium ordered fraction and ordered positions within InGaAs QDs by XSW}

Figure 1 shows the indium $L \alpha$ fluorescence yield $Y(\theta)$ and corresponding reflectivity $R(\theta)$ as a function of incident angle $\theta$ for the eight symmetry-inequivalent $h k l$ Bragg reflections measured in each sample. The reflectivity $R(\theta)$ and normalized fluorescence yield $Y(\theta)$ profiles are analyzed based on the dynamical diffraction theory (DDT) for a perfect single crystal $[25,26]$ (refer to the SM for details [22]) with coherent fraction $\left(f_{\mathrm{H}}\right)$ and position $\left(P_{\mathrm{H}}\right)$ as fitting parameters using the equation $[27,28]$

$$
Y(\theta)=1+R(\theta)+2 \sqrt{R(\theta)} f_{\mathrm{H}} \cos \left[v(\theta)-2 \pi P_{\mathrm{H}}\right],
$$

where $v(\theta)$ is the XSW phase obtained from DDT calculations along with the measured reflectivity $R(\theta)$. The fitting parameters $f_{\mathrm{H}}$ and $P_{\mathrm{H}}$ obtained from this model independent analysis of Bragg reflections for both the samples are also shown in Table I. Uncertainties in the model independent parameters ( $f$ and $P$ ) are primarily due to counting statistics in the XRF yield values, errors in using DDT on a slightly nonperfect single crystal, and gradient of $\chi^{2}$ during the fitting of Eq. (2) to the data. If In atoms are laterally restricted to $\mathrm{Ga} x, y$ coordinates and only allowed to be displaced in the $z$ direction then measured $P_{\mathrm{H}}$ values with the same $l$ indices would be equivalent to within the \pm 0.03 uncertainty for each sample. Positional shifts of indium atoms from the ideal substratelike $\mathrm{Ga}$ atomic plane in both the (004) and (111) directions can be determined by $d_{004} P_{004}$ and $d_{111} P_{111}$. These are the measured displacements for the distribution of In atoms with respect to 


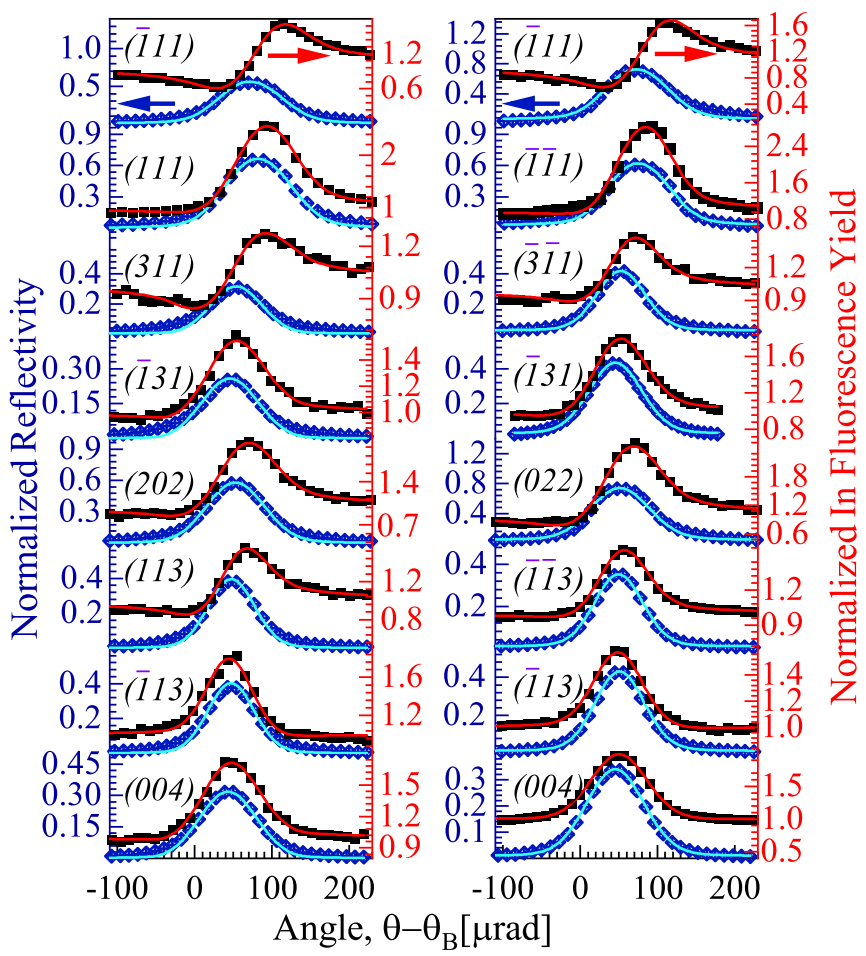

FIG. 1. Reflectivity $R(\theta)$ and normalized indium $L \alpha$ fluorescence yield $Y(\theta)$ as a function of x-ray incident angle. Left: 50$\AA$ GaAs cap InGaAs-QDs/GaAs (001); right: $150-\AA$ GaAs cap InGaAs-QDs/GaAs(001) for measured Bragg reflections as indicated above for each subfigure. The solid blue and red lines are best fits to the experimental data (symbols) of the reflectivity and fluorescence yield, respectively.

bulklike Ga (004) and (111) planes. Note that $P$ and $f$ are not direct measurements for one In atomic layer, but are related to the distribution created by the projection of all fluorescent In atoms into one primitive unit cell of the GaAs substrate.

The indium coherent positions for the (004) Bragg reflection are determined as $P_{004}=0.197 \pm 0.03$ for sample A and $P_{004}=0.262 \pm 0.03$ for sample B (refer to Table I), which locate the mean heights of indium atoms to be $h=$ $0.28 \pm 0.04 \AA$ and $0.37 \pm 0.04 \AA$, respectively. These results can be directly compared to three earlier case studies for MBE of 1-ML InAs on GaAs(001) that only measured the (004) XSW $f$ and $P$ values. For the case with a $25-\AA ̊ \AA$ cap, Lee et al. [29] found $h=0.22 \AA$ and $f_{004}=0.43$, with a 100 - $\AA$ cap, Lee et al. [30] found $h=0.24 \AA$ and $f_{004}=0.67$, and for the case of a 300-A cap Giannini et al. [31] found $h=0.25 \AA$ and $f_{004}=0.58$. Woicik et al. carried out extended x-ray- absorption fine-structure [32] and anomalous x-ray scattering [33] studies for these 1-ML cases. Our In mean height values are slightly higher than these 1-ML cases, most likely because our thicker samples of 1.7 and 3.5 MLs both formed QD islands with strained multiple layers. Here, it should be noted that Stranski-Krastanov mode [20,34] QDs are only formed on $\operatorname{GaAs}(001)$ substrates when the deposited InAs is greater than 1 ML [35]. Thus, the referenced 1-ML results are from 2D thin films on GaAs(001) while our XSW study is on QDs.

We performed (111) and (111) XSW measurements where the coherent positions have their origins at the substrate $\mathrm{Ga}$ lattice position. The GaAs (111) bilayer polarity is inverted as we switch between the [111] and [1111] directions (see the SM, Fig. S5 [22]) which leads to the dissimilarity in the modulation of the In yield exhibited between (111) and (111) Bragg reflections (see Fig. 1). $P_{111}$ and $P_{\overline{111}}$ are found to be 0.034 and 0.028 for sample $\mathrm{A}$ and 0.03 and 0.05 for sample B, respectively (Table I). Since the (111) and (111) reflections measure the $[110]$ and $[\overline{1} 10]$ components of the in-plane atom distribution, the clear discrepancy between the measured values of $f_{111}(0.82$ for sample $\mathrm{A}$ and 0.78 for sample B) and of $f_{\overline{1} 11}$ (0.61 for sample A and 0.72 for sample B) indicates an anisotropic lateral indium distribution within the QDs; i.e., the In distribution width is wider along the [ $[\overline{1} 10]$ than along the [110] directions. In addition to the experimental optical anisotropic property observation $[15,16]$ as a function of in-plane direction measurements, there are also some theoretical studies [36] to understand the effect of the anisotropy on the many electron ground state [37] and other electronic properties [38]. The electronic and optical anisotropies are directly linked to the potential produced by the anisotropic distribution of composite material atoms within the QDs. Our result supports the anisotropy reported in the previous studies of the initial growth stage [39] of lattice-mismatched III-V heteroepitaxial structures. However, all the earlier studies are model dependent with the assumption of either circular, cylindrical, or other simple distributions $[3,36,38]$.

The results of XSW measurements for the (311), (131), (113), (113) Bragg reflections for the 50- $\AA$ capped sample A show indium atom positions are downward shifted by $0.024 \pm$ $0.03 \AA$ along the [131] direction, but in the [311] direction indium atoms are upward shifted by $0.036 \pm 0.03 \AA$ from the unstrained Ga atom position (Table I). For the 150- $\AA$ capped sample $\mathrm{B}$, indium atoms are displaced upward significantly along the [1 131$]$ and [311] directions by $0.073 \pm 0.03 \AA$ and $0.17 \pm 0.03 \AA$, respectively. The relation of the magnitudes of the coherent fractions $f_{111}>f_{1 \overline{1} 1}$ is in good agreement with $f_{311}>f_{\overline{1} 31}$ for sample $\mathrm{B}$, but this is not the case for sample A $\left(f_{311}<f_{\overline{1} 31}\right.$ although $\left.f_{111}>f_{1 \overline{1} 1}\right)$. However, it should be

TABLE I. XSW model-independent values for In coherent fraction $\left(f_{\mathrm{H}}\right)$ and coherent position $\left(P_{\mathrm{H}}\right)$ from $\chi^{2}$ fits of Eq. (2) to the data in Fig. 1. As a reference $P_{\mathrm{H}}=0, f_{111}=0.99, f_{311}=0.97, f_{202}=0.97$, and $f_{004}=0.95$, for ideal substrate Ga atom positions.

\begin{tabular}{|c|c|c|c|c|c|c|c|c|c|}
\hline Sample & (hkl) & $\overline{1} 11$ & 111 & 311 & $\overline{1} 31$ & 022 & 113 & $\overline{1} 13$ & 004 \\
\hline \multirow[t]{2}{*}{ 50-Å GaAs cap InGaAs QDs/GaAs(001) } & $f_{\mathrm{H}}( \pm 0.03)$ & 0.82 & 0.61 & 0.73 & 0.34 & 0.58 & 0.61 & 0.44 & 0.44 \\
\hline & $P_{\mathrm{H}}( \pm 0.03)$ & 0.034 & 0.028 & 0.021 & -0.014 & 0.069 & 0.121 & 0.169 & 0.197 \\
\hline \multirow[t]{2}{*}{ 150-Å GaAs cap InGaAs QDs/GaAs(001) } & $f_{\mathrm{H}}( \pm 0.03)$ & 0.78 & 0.72 & 0.52 & 0.66 & 0.93 & 0.25 & 0.2 & 0.79 \\
\hline & $P_{\mathrm{H}}( \pm 0.03)$ & 0.03 & 0.053 & 0.10 & 0.043 & 0.116 & 0.216 & 0.186 & 0.262 \\
\hline
\end{tabular}


noted that the (311) and (131) in-plane components are not along the [110] and [111] directions. This clearly indicates that simplified models are not suitable for understanding the anisotropic properties of QDs. $f$ values for (311) and (131) are more sensitive to the in-plane anisotropy as they have stronger in-plane components and much smaller $d$ spacing.

Coherent positions $\left(P_{\mathrm{H}}\right)$ for (113) and (113) are found to be 0.121 and 0.169 for sample $A$, which increase to 0.216 and 0.186 respectively for sample B (Table I). Moreover, $f_{\mathrm{H}}$ of 0.61 and 0.44 for (113) and (113) for sample A are diminished to $f_{\mathrm{H}}=0.22$ and 0.19 , respectively, indicating an enhancement of indium atom distribution along [113] and [113] directions upon thicker GaAs cap layer growth [40] over QDs. This result indicates that (113) and (113) are the most affected Bragg peaks when the GaAs cap layer thickness increases.

In addition, (022) XSW results show indium $P_{022}$ to be $0.069 \pm 0.03$ and $0.116 \pm 0.03$ for sample A and sample B (see Table I) indicating that indium atoms are upward shifted along the [011] direction with respect to $\mathrm{Ga}$ atom positions within the GaAs unit cell. This result supports previous results about the elongation of QDs along [011] directions by microscopy and GID measurements [41].

\section{B. XSW direct space atomic-density map of QDs}

A direct space atomic-density map can be generated from a sufficient number of XSW measured symmetry inequivalent Fourier components in reciprocal space by Fourier inversion using the equation $[13,27,42,43]$

$$
\begin{aligned}
\rho(r) & =\sum_{\mathrm{H}} f_{\mathrm{H}} \exp \left[-2 \pi i\left(H \cdot r-P_{\mathrm{H}}\right)\right] \\
& =1+2 \sum_{\mathrm{H} \neq-\mathrm{H}, \mathrm{H} \neq 0} f_{\mathrm{H}} \cos \left[2 \pi\left(H \cdot r-P_{\mathrm{H}}\right)\right] .
\end{aligned}
$$

The resultant 3D atomic density maps for samples A are shown in Fig. 2. The generated maps are independent of any presupposed structural constraints and only assume a GaAs FCC zinc-blende substrate lattice with Ga located at the origin (corner) of the nonprimitive cubic unit cell. Indium atoms occupy the face-centered positions [Figs. 2(a) and 2(d)] within the QDs as expected for InGaAs alloy formation during epitaxial growth. Indium atoms show periodicity equivalent to the substrate rhombohedral primitive unit cell [Fig. 2(c)] due to the Fourier summation process. Indium density maxima are vertically upward shifted with respect to the fcc $\mathrm{Ga}$ atom positions. A 2D cut of the (100) plane [Figs. 2(b) and 2(e)] reveals quantitative amounts of $0.04 a_{\mathrm{GaAs}}$ and $0.06 a_{\mathrm{GaAs}}$ outward shifting of indium atoms from Ga sites. 1D line cuts along three vertical lines towards [001] are drawn at real space $(x, y)$ coordinates of $(0,0),(0.25,0.25)$, and $(0,0.5)$ of the unit cell [Figs. 2(c) and 2(f)] with indium locations at the corner of the cube for sample A and sample B, respectively. Each peak for indium atomic density shows finite full width at half maximum (FWHM) as the summation is resolution limited to $\left(d_{004} / a\right) / 2=0.125$ due to the Fourier series truncation effect limited by a finite number of measured $h k l$ Fourier components. Therefore, the average out-of-plane lattice parameters (a)

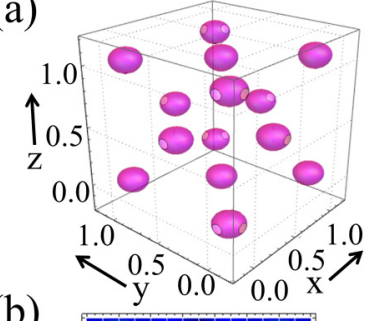

(d)

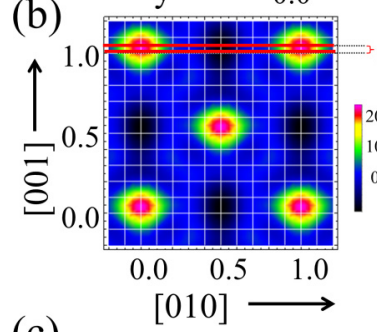

(e)

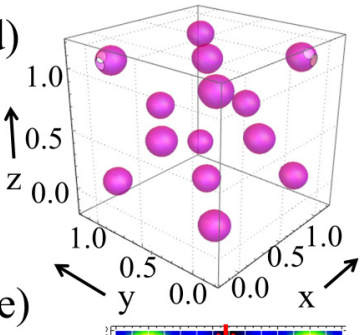

(c) $0.04 \mathrm{a}_{\mathrm{GaAs}}$

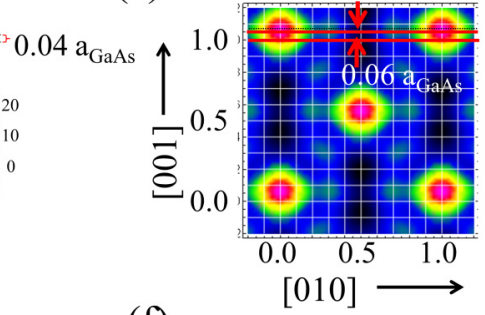

(f)

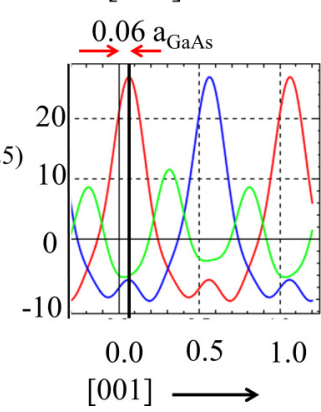

$(\mathrm{x}, \mathrm{y})$

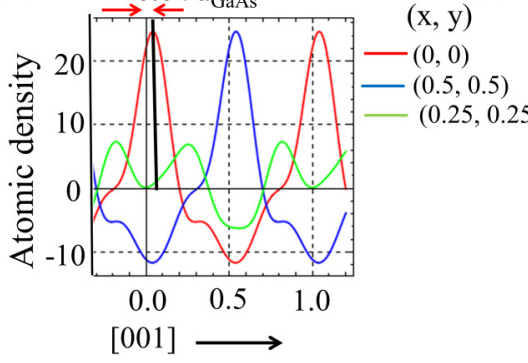

$[001]$

FIG. 2. (a), (d) XSW-measured model-independent 3D indium atomic map for (a) 50- $\AA$-capped and (d) 150- $\AA$-capped InGaAsQDs/GaAs(001). The outlined black cube is larger than the GaAs unit cell to demonstrate the indium atom positions. The $x, y$, and $z$ axes are in units of fractional GaAs unit-cell lattice vectors $a, b$, and $c$ with the origin placed at the Ga site. (b), (e) Atomic density of indium on the (100) plane located at $x=0$ of the GaAs unit cell for (b) 50- $\AA$-capped and (e) 150- $\AA$-capped QDs sample. Five red spots denote indium atom positions at expected fcc sites. Due to strain the indium atoms are outwardly shifted from the $\mathrm{Ga}$ atom positions of the GaAs weighted unit cell. (c), (f) In 3D density plotted along the $c$ axis to produce a $1 \mathrm{D}$ In atomic density as a function of the fractional $c$-axis coordinate $z$ for the GaAs unit cell for (c) sample A and (f) sample B. Red, blue, green lines are along $(0,0, z),(0,0.5, z)$, and $(0.25,0.25, z)$, respectively.

of the QDs can be estimated to be 5.88 and $5.99 \AA$ for sample $\mathrm{A}$ and sample $\mathrm{B}$, respectively.

\section{Numerical calculations for ordered fractions and ordered positions of QD atoms based on a GID-derived model}

We have numerically calculated the ordered fraction and ordered position of the indium atoms within the QDs using GID-obtained structural parameters. Details of the GID method to measure the composition and lateral width as a function of height within the QDs can be found in Ref. [19]. For the development of the atomic-imaging technique demonstrated here, we have used the results of these representative samples A and B. Initially ignoring strain effects, we have considered two sublayers of $\mathrm{In} / \mathrm{Ga}$ and As atoms separated by $d_{002}$ of GaAs $(\approx 2.82 \AA)$ as indicated in Figs. 3(a) and 3(d). Sample A is represented by 13 such In/Ga atomic sublayers 


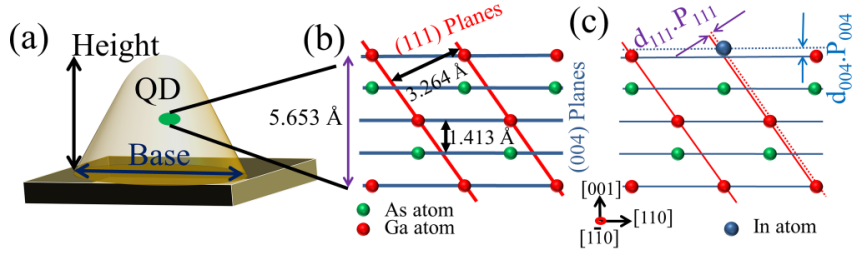

(d)

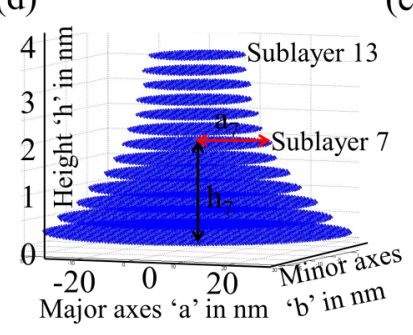

(e)

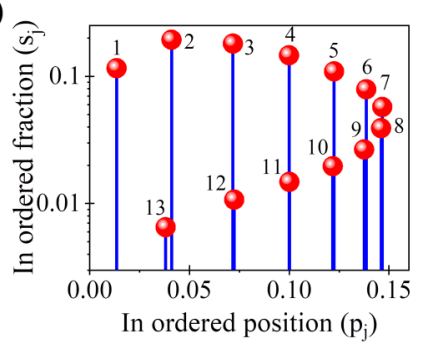

(f)

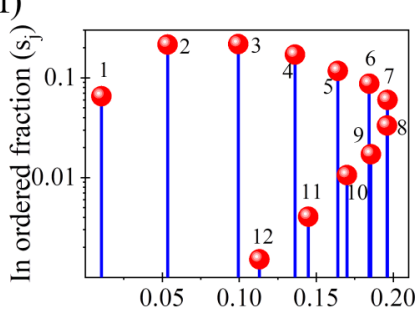

In ordered position $\left(\mathrm{p}_{\mathrm{j}}\right)$ (g)

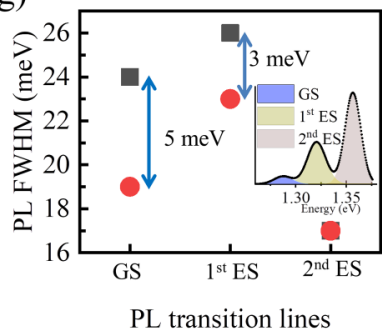

FIG. 3. (a) Schematic sketch of an average GaAs cap InGaAsQDs/GaAs(001). (b) Unstrained GaAs atomic arrangement showing atoms Ga (red circle) and As (green circle) along with (004) and (111) bulk planes as blue and red lines, respectively. (c) Illustration showing how the XSW measured values for $P_{004}$ and $P_{111}$ are used to triangulate the average In atomic (blue dot) position relative to the unstrained substrate GaAs atomic arrangement. (d) The 50- $\AA$ GaAs capped QD is segmented into $13 \mathrm{In} / \mathrm{Ga}$ atomic sublayers (blue ellipsoidal platelets). Sublayers 7 and 13 are shown for reference. Here, $a_{7}$ and $h_{7}$ denotes the major axis dimension and height of the seventh sublayer of the GaAs substrate. (e), (f) Ordered fractions $\left(s_{\mathrm{j}}\right)$ and out-of-plane ordered positions $\left(p_{\mathrm{j}}\right)$ of the 13 indium atomic sublayers for (e) sample A and the 12 indium atomic sublayers for (f) sample B with reference to unstrained GaAs unit cell. The numbers indicate the sublayer numbers. (g) Reduction of FWHM of ground, first and second excited-state PL transition lines indicated by the blue arrow. PL spectra for sample B is shown in the inset for reference.

$\left[(13-1) \times\left(d_{(002, \mathrm{GaAs})} \approx 2.82 \AA\right)=33.94 \AA\right]$ as indicated in Fig. 3(d). The in-plane extent of the sublayers representing an average QD is also shown in Fig. 3(d). Each sublayer is approximated to be elliptical in shape as observed [19] in GID and AFM measurements (refer to Fig. S3 in the SM [22]). The indium content in each such sublayer is calculated from the area $(\pi a b)$ covered by an elliptical disk multiplied by the In concentration $(x)$ in the corresponding sublayer. Finally, the ordered indium fractions $\left(s_{\mathfrak{j}}\right)$ for each sublayer are defined as

$$
s_{\mathrm{j}}=\frac{x_{\mathrm{j}} a_{\mathrm{j}} b_{\mathrm{j}}}{\sum_{j=1}^{n} x_{\mathrm{j}} a_{\mathrm{j}} b_{\mathrm{j}}},
$$

where $j$ indicates a particular atomic sublayer. It should be noted here that $\mathrm{In} / \mathrm{Ga}$ atomic sublayer positions can be shifted from the unstrained $\mathrm{Ga}$ atomic positions [refer to Figs. 3(b)

and 3(c)] due to the variation of biaxial strain throughout the height of a QD and this shift can be determined with the method presented here. As the out-of plane diffraction from a single layer of the QD cannot be measured directly, the out-of-plane strain for each sublayer is calculated from the in-plane strain measured through GID for In composition $(x)$ dependent stress tensors as

$$
a_{\perp}=\frac{-2 C_{12}(x)}{C_{11}(x)} a_{\|} .
$$

The actual In/Ga atomic positions for each sublayer are then determined by the differences between the out-of-plane lattice parameter with that of the substrate as

$$
\Delta a_{\mathrm{j}}=\left|a_{\perp j}-a_{\mathrm{GaAs}}\right| .
$$

For example, the second atomic layer position with respect to the unstrained $\mathrm{Ga}$ atomic sublayer can be determined by

$$
\Delta_{2}=\sum_{i=1}^{2} \Delta a_{i}
$$

and the third atomic sublayer position shift can be estimated by

$$
\Delta_{3}=\sum_{i=1}^{3} \Delta a_{i} .
$$

The accumulated shift $\left(\Delta_{\mathrm{j}}\right)$ for the $j$ th sublayer scaled by $d_{(002, \mathrm{GaAs})}$ is called the ordered position $p_{\mathrm{j}}$ as

$$
p_{\mathrm{j}}=\frac{\Delta_{\mathrm{j}}}{d_{(002, \mathrm{GaAs})}} .
$$

Indium ordered fractions $\left(s_{\mathrm{j}}\right)$ at the actual ordered positions $\left(p_{\mathrm{j}}\right)$ for samples A and B are shown in Figs. 3(e) and 3(f). A further assumption of isostrain distribution of indium atoms in the in-plane direction makes $F(l, n)$ [see Eq. (1)] independent of $h$ and $k$ Miller indices. Hence the Bragg reflections having the same $l$ value generate identical $P_{\mathrm{H}}$ values. For example, (111), ( $(\overline{1} 11)(\overline{1} 31),(\overline{3} \overline{1} 1)$, and (131) Bragg reflections give identical $P_{\mathrm{H}}$ of 0.042 and 0.058 for sample A and B, respectively. This kind of in-plane isostrain assumption is commonly used during modeling of GID results. All the $P_{\mathrm{H}}$ and $f_{\mathrm{H}}$ values obtained from the elliptical-shaped GID model are tabulated in Table II for both samples.

The GID-model dependent calculations of coherent fraction $f_{\mathrm{H}}$ and coherent position $P_{\mathrm{H}}$ data clearly show an upward shift of the indium atoms from the nearest As atoms by 0.238 $\AA$ for sample $\mathrm{A}$ and $0.332 \AA$ for sample B with corresponding $P_{004}$ values of 0.169 and 0.235 , respectively. The indium atoms are shifted more in the upward direction in sample B due to the additional strain imposed by the higher thickness of the GaAs cap layer. This is in agreement with the results obtained from XSW, but the $f_{004}$ are always restricted to lower values $(\sim 0.5)$ and cannot sense the higher indium ordering for sample B. This restriction comes due to the assumption of the isostrain in plane which constrains indium atoms to sit at fixed ordering in the in-plane direction forcing them to lower ordering in the out-of-plane direction to compensate. As a consequence it is expected that GID would give higher in-plane indium ordering in this model. As expected, 
TABLE II. Coherent fractions $\left(f_{\mathrm{H}}\right)$ and coherent positions $\left(P_{\mathrm{H}}\right)$ from GID model-dependent calculations. As a reference $P_{\mathrm{H}}=0, f_{111}=$ $0.99, f_{311}=0.97, f_{202}=0.97$, and $f_{004}=0.95$, for ideal substrate $\mathrm{Ga}$ atom positions.

\begin{tabular}{|c|c|c|c|c|c|c|c|c|c|}
\hline Sample & $(h k l)$ & $\overline{1} 11$ & 111 & 311 & $\overline{1} 31$ & 022 & 113 & $\overline{1} 13$ & 004 \\
\hline \multirow[t]{2}{*}{ 50-Å GaAs cap InGaAs QDs/GaAs(001) } & $f_{\mathrm{H}}( \pm 0.05)$ & 0.9 & 0.9 & 0.7 & 0.7 & 0.74 & 0.65 & 0.65 & 0.52 \\
\hline & $P_{\mathrm{H}}( \pm 0.05)$ & 0.042 & 0.042 & 0.042 & 0.042 & 0.084 & 0.126 & 0.126 & 0.169 \\
\hline \multirow[t]{2}{*}{ 150-Å GaAs cap InGaAs QDs/GaAs(001) } & $f_{\mathrm{H}}( \pm 0.05)$ & 0.89 & 0.89 & 0.69 & 0.69 & 0.73 & 0.61 & 0.61 & 0.47 \\
\hline & $P_{\mathrm{H}}( \pm 0.05)$ & 0.058 & 0.058 & 0.058 & 0.058 & 0.117 & 0.175 & 0.175 & 0.235 \\
\hline
\end{tabular}

disagreement on the $f_{\mathrm{H}}$ appears as GID predicts higher $f_{\mathrm{H}}$ 0.65 (sample A) and 0.61 (sample B) for the most in-plane sensitive (113) and (113) Bragg reflections. The (113) and (113) Bragg reflections (Table II) exhibit a $P_{\mathrm{H}}$ of 0.126 for the 50- $\AA$ capped sample $A$ and an increased value of 0.175 for the $150-\AA$ capped sample $\mathrm{B}$. The obtained $f_{\mathrm{H}}$ is 0.9 for (111) and (111), 0.7 for (311) and (131), and 0.65 for (113) and (113) Bragg reflections for sample A (Table II). $P_{111}$ values of 0.04 and 0.05 for sample A and sample B show a reasonable agreement with the XSW obtained $P_{\mathrm{H}}$ values. Uncertainties in $f$ and $P$ values arise due to the model fitting of GID data.

\section{Photoluminescence characteristics}

We have studied the effect of the indium ordering distribution on the PL linewidth for these two samples. The ground-state (GS) transition line $\left(e_{\mathrm{GS}}-h h_{\mathrm{GS}}\right)$ and the first excited-state (first ES) transition line $\left(e_{1}-h h_{1}\right)$ are observed at 1289 and $1322 \mathrm{meV}$ respectively for the $150-\AA$ capped QD sample [inset in Fig. 3(g)]. The FWHMs are found to be 24 and $26 \mathrm{meV}$ for the GS and the first ES state transition lines respectively for the $50-\AA$ capped QD as shown by the black dots in Fig. 3(g). Model-independent fitting of the XSW profiles of the InGaAs QDs reveals that the coherent fraction $f_{\mathrm{H}}$ of both (022) and (004) Bragg reflections are increased significantly for the $150-\AA$ GaAs capped as compared to the 50- $\AA$ GaAs capped QD layer (Table I), indicating more coherence of the QD in the [001] and [011] directions with 150 capping. As a result, the PL FWHMs were observed as 19 and $23 \mathrm{meV}$ for the GS and the first ES transition lines respectively for the $150-\AA$ capped QD. Reduction of $5 \mathrm{meV}$ in the GS and $3 \mathrm{meV}$ in the first ES PL FWHM [refer to Fig. 3(g)] is therefore observed when indium is $80-90 \%$ coherently ordered (sample B) in the out-of-plane direction rather than the $45-60 \%$ coherently ordered for sample A. The reduction in FWHM of the GS of the PL line is about $22 \%$ when coherent indium ordering is doubled (sample B) in the out-of-plane direction.

\section{CONCLUSIONS}

In conclusion, the present study provides a comprehensive picture of the microscopic and macroscopic structure of InGaAs QDs and the change in structure due to variation of GaAs cap-layer thickness. Our results clearly show that the QD's unit cell becomes more elongated along the [001] direction with increasing GaAs cap-layer thickness due to excess strain introduced by the GaAs matrix into the QDs and also illustrates higher anisotropic growth of the QDs in the in-plane directions. These features are attributed to the intriguing and subtle interplay between the In-As bond elongation due to the strain introduced by the substrate and cap GaAs. The macroscopic quantum dot structure is mapped into a microscopic unit cell by GID for comparison with the information obtained by XSW techniques. Disagreements between coherent fractions and coherent positions of indium atoms for in-plane sensitive Bragg reflections are due to the limitation of isostrain in-plane modeling based on GID measurements. The anisotropic optical and electronic properties of these types of QDs can be understood with these structural studies which directly probe structural in-plane anisotropy within QDs. Our study clearly indicates that (113) and (113) Bragg peaks are more affected and sensitive compared with the (022) and (004) with GaAs cap thickness. We recommend continued focus on both (113) and (113) Bragg peaks during further study of the effect of cap layer thickness on QDs. Our study demonstrates that the FWHM of PL lines is directly related to the ordering of In atoms in the out-of-plane direction; more ordering along [001] and [011] directions produces sharper PL lines. Similar studies are anticipated for the correlation between structure and optoelectronic properties of other nanostructures important for devices like QD-based laser and LED displays. Moreover, these types of measurements of constituent atom positions and orderings in all possible Bragg directions will provide vital information about anisotropy which will in turn help others to model the resultant anisotropic optical and electronic properties displayed by these QDs.

\section{ACKNOWLEDGMENTS}

We thank the Indo-US Science and Technology Forum (IUSSTF) for their financial support for doing experiments under the project "Crystallization at Interfaces." This work was partially supported at Northwestern University (NU) by the Institute for Catalysis in Energy Processes (ICEP) under U.S. DOE Grant No. DE-FG02-03ER15457 and National Science Foundation MRSEC (Grant No. DMR-1720139). GID experiments at PETRA-III synchrotron DESY were carried out through the India-DESY collaboration program. XSW experiments were performed at the Argonne National Lab (ANL) Advanced Photon Source (APS) DND-CAT 5ID$\mathrm{C}$ station. ANL is supported by the DOE under Grant No. DE-AC02-06CH11357. DND-CAT is supported through E. I. DuPont de Nemours \& Co., Northwestern University, The Dow Chemical Co., and the NSF funded MRSEC at NU. Preliminary and calibration measurements made use of the NU X-ray Diffraction Facility supported by the MRSEC. 
[1] D. N. Basov, R. D. Averitt, and D. Hsieh, Towards properties on demand in quantum materials, Nat. Mater. 16, 1077 (2017).

[2] V. I. Klimov, A. A. Mikhailovsky, S. Xu, A. Malko, J. A. Hollingsworth, C. A. Leatherdale, H. J. Eisler, and M. G. Bawendi, Optical gain and stimulated emission in nanocrystal quantum dots, Science 290, 314 (2000).

[3] M. Sharma, M. K. Sanyal, I. Farrer, D. A. Ritchie, A. B. Dey, A. Bhattacharyya, O. H. Seeck, J. Skiba-Szymanska, M. Felle, A. J. Bennett, and A. J. Shields, Density dependent composition of InAs quantum dots extracted from grazing incidence x-ray diffraction measurements, Sci. Rep. 5, 15732 (2015).

[4] P. M. Petroff, Semiconductor self-assembled quantum dots: Present status and future trends, Adv. Mater. 23, 2372 (2011).

[5] X. Dai, Y. Deng, X. Peng, and Y. Jin, Quantum-dot lightemitting diodes for large-area displays: Towards the dawn of commercialization, Adv. Mater. 29, 1607022 (2017).

[6] S. Chen, M. Tang, Q. Jiang, J. Wu, V. G. Dorogan, M. Benamara, Y. I. Mazur, G. J. Salamo, P. Smowton, A. Seeds, and $\mathrm{H}$. Liu, InAs/GaAs quantum-dot superluminescent lightemitting diode monolithically grown on a Si substrate, ACS Photon. 1, 638 (2014).

[7] S. Fafard, K. Hinzer, S. Raymond, M. Dion, J. McCaffrey, Y. Feng, and S. Charbonneau, Red-emitting semiconductor quantum dot lasers, Science 274, 1350 (1996).

[8] Z. Yuan, B. E. Kardynal, R. M. Stevenson, A. J. Shields, C. J. Lobo, K. Cooper, N. S. Beattie, D. A. Ritchie, and M. Pepper, Electrically driven single-photon source, Science 295, 102 (2001).

[9] P. Lodahl, S. Mahmoodian, and S. Stobbe, Interfacing single photons and single quantum dots with photonic nanostructures, Rev. Mod. Phys. 87, 347 (2015).

[10] K. A. Moler, Imaging quantum materials, Nat. Mater. 16, 1049 (2017).

[11] C. Song, R. Bergstrom, D. Ramunno-Johnson, H. Jiang, D. Paterson, M. D. de Jonge, I. McNulty, J. Lee, K. L. Wang, and J. Miao, Nanoscale Imaging of Buried Structures with Elemental Specificity using Resonant X-Ray Diffraction Microscopy, Phys. Rev. Lett. 100, 025504 (2008).

[12] I. Robinson and R. Harder, Coherent x-ray diffraction imaging of strain at the nanoscale, Nat. Mater. 8, 291 (2009).

[13] Z. Feng, A. Kazimirov, and M. J. Bedzyk, Atomic imaging of oxide-supported metallic nanocrystals, ACS Nano 5, 9755 (2011).

[14] W. Q. Ma, M. L. Hussein, J. L. Shultz, G. J. Salamo, T. D. Mishima, and M. B. Johnson, Enhancing the in-plane spatial ordering of quantum dots, Phys. Rev. B 69, 233312 (2004).

[15] H. Tahara, Y. Ogawa, F. Minami, K. Akahane, and M. Sasaki, Anisotropic optical properties of excitons in strain-controlled InAs quantum dots, Phys. Rev. B 87, 035304 (2013).

[16] D. G. Cooke, F. A. Hegmann, Y. I. Mazur, W. Q. Ma, X. Wang, Z. M. Wang, G. J. Salamo, M. Xiao, T. D. Mishima, and M. B. Johnson, Anisotropic photoconductivity of InGaAs quantum dot chains measured by terahertz pulse spectroscopy, Appl. Phys. Lett. 85, 3839 (2004).

[17] A. Singha, V. Pellegrini, S. Kalliakos, B. Karmakar, A. Pinczuk, L. N. Pfeiffer, and K. W. West, Optical anisotropy of electronic excitations in elliptical quantum dots, Appl. Phys. Lett. 94, 073114 (2009).

[18] T. Kiessling, A. V. Platonov, G. V. Astakhov, T. Slobodskyy, S. Mahapatra, W. Ossau, G. Schmidt, K. Brunner, and L. W.
Molenkamp, Anomalous in-plane magneto-optical anisotropy of self-assembled quantum dots, Phys. Rev. B 74, 041301(R) (2006).

[19] A. B. Dey, M. K. Sanyal, I. Farrer, K. Perumal, D. A. Ritchie, Q. Li, J. Wu, and V. Dravid, Correlating photoluminescence and structural properties of uncapped and GaAs-capped epitaxial InGaAs quantum dots, Sci. Rep. 8, 7514 (2018).

[20] A. Baskaran and P. Smereka, Mechanisms of stranski-krastanov growth, J. Appl. Phys. 111, 044321 (2012).

[21] J. Tersoff, C. Teichert, and M. G. Lagally, Self-Organization in Growth of Quantum Dot Superlattices, Phys. Rev. Lett. 76, 1675 (1996).

[22] See Supplemental Material at http://link.aps.org/supplemental/ 10.1103/PhysRevMaterials.4.056002 for experiment section: InGaAs QDs/GaAs(001) growth by molecular beam epitaxy, cross-sectional transmission electron microscopy, atomicforce microscopy, and photoluminescence studies on InGaAsQDs/GaAs(001) TR-XRF calibrated by RBS to determine in coverage in InGaAs-QDs/GaAs total reflection $\mathrm{x}$-ray fluorescence to calculate GaAs capping layer thickness over InGaAsQDs; data analysis section: characterization of GaAs-capped InGaAs-QDs/GaAs(001) by XSW, grazing incidence (GID) measurements to calculate height base composition and strain of InGaAs-QDs.

[23] R. S. Becker, J. A. Golovchenko, and J. R. Patel, X-Ray Evanescent-Wave Absorption and Emission, Phys. Rev. Lett. 50, 153 (1983).

[24] A. Chellu, Optical properties of close-to-surface self-assembled InAs/GaAs quantum dots, (2018).

[25] B. W. Batterman and H. Cole, Dynamical diffraction of X rays by perfect crystals, Rev. Mod. Phys. 36, 681 (1964).

[26] P. L. Cowan, J. A. Golovchenko, and M. F. Robbins, X-Ray Standing Waves at Crystal Surfaces, Phys. Rev. Lett. 44, 1680 (1980).

[27] M. J. Bedzyk and L. Cheng, X-ray standing wave studies of minerals and mineral surfaces: Principles and applications, Rev. Mineral. Geochem. 49, 221 (2002).

[28] J. Zegenhagen, Surface structure determination with x-ray standing waves, Surf. Sci. Rep. 18, 202 (1993).

[29] T.-L. Lee, Y. Qian, P. Lyman, J. Woicik, J. Pellegrino, and M. Bedzyk, The use of $\mathrm{x}$-ray standing waves and evanescent-wave emission to study buried strained-layer heterostructures, Phys. B (Amsterdam, Neth.) 221, 437 (1996).

[30] T.-L. Lee, M. R. Pillai, J. C. Woicik, G. Labanda, P. F. Lyman, S. A. Barnett, and M. J. Bedzyk, Atomic-resolution study of lattice distortions of buried $\operatorname{In}_{x} \mathrm{Ga}_{1-x}$ As monolayers in GaAs(001), Phys. Rev. B 60, 13612 (1999).

[31] C. Giannini, L. Tapfer, S. Lagomarsino, J. C. Boulliard, A. Taccoen, B. Capelle, M. Ilg, O. Brandt, and K. H. Ploog, Phys. Rev. B 48, 11496(R) (1993).

[32] J. C. Woicik, Random-cluster calculation of bond lengths in strained-semiconductor alloys, Phys. Rev. B 57, 6266 (1998).

[33] J. C. Woicik, J. O. Cross, C. E. Bouldin, B. Ravel, J. G. Pellegrino, B. Steiner, S. G. Bompadre, L. B. Sorensen, K. E. Miyano, and J. P. Kirkland, Diffraction anomalous finestructure study of strained $\mathrm{Ga}_{1-x} \operatorname{In}_{x}$ As on GaAs(001), Phys. Rev. B 58, R4215(R) (1998).

[34] P. M. Petroff and S. P. DenBaars, MBE and MOCVD growth and properties of self-assembling quantum dot arrays in 
III-V semiconductor structures, Superlattices Microstruct. 15, 15 (1994).

[35] J. M. García, J. P. Silveira, and F. Briones, Strain relaxation and segregation effects during self-assembled InAs quantum dots formation on $\operatorname{GaAs}(001)$, Appl. Phys. Lett. 77, 409 (2000).

[36] L. H. Friedman, Anisotropy and order of epitaxial self-assembled quantum dots, Phys. Rev. B 75, 193302 (2007).

[37] M. Fujito, A. Natori, and H. Yasunaga, Many-electron ground states in anisotropic parabolic quantum dots, Phys. Rev. B 53, 9952 (1996).

[38] A. V. Madhav and T. Chakraborty, Electronic properties of anisotropic quantum dots in a magnetic field, Phys. Rev. B 49, 8163 (1994).

[39] M. V. Belousov, N. N. Ledentsov, M. V. Maximov, P. D. Wang, I. N. Yasievich, N. N. Faleev, I. A. Kozin, V. M. Ustinov,
P. S. Kop'ev, and C. M. Sotomayor Torres, Energy levels and exciton oscillator strength in submonolayer InAs-GaAs heterostructures, Phys. Rev. B 51, 14346 (1995).

[40] R. Songmuang, S. Kiravittaya, and O. G. Schmidt, Shape evolution of InAs quantum dots during overgrowth, J. Cryst. Growth 249, 416 (2003).

[41] G. Costantini, A. Rastelli, C. Manzano, P. Acosta-Diaz, R. Songmuang, G. Katsaros, O. G. Schmidt, and K. Kern, Interplay Between Thermodynamics and Kinetics in the Capping of InAs/GaAs(001) Quantum Dots, Phys. Rev. Lett. 96, 226106 (2006).

[42] L. Cheng, P. Fenter, M. J. Bedzyk, and N. C. Sturchio, FourierExpansion Solution of Atom Distributions in a Crystal Using X-Ray Standing Waves, Phys. Rev. Lett. 90, 255503 (2003).

[43] J. Zegenhagen and A. Kazimirov, The X-Ray Standing Wave Technique, Series on Synchrotron Radiation Techniques and Applications (World Scientific, Singapore, 2011), Vol. 7. 